\title{
Numerical study on the fracture characteristics and failure mode of hard coal under coupled static and dynamic loads
}

\author{
Lihai Tan ${ }^{1}$, Ting Ren ${ }^{1, *}$, Xiaohan Yang ${ }^{1, \dagger}$ and Xueqiu $\mathrm{He}^{2}$ \\ ${ }^{1}$ School of Civil, Mining and Environmental Engineering, University of Wollongong, NSW 2522, \\ Australia \\ ${ }^{2}$ School of Civil and Environmental Engineering, University of Science and Technology, Beijing \\ 100083, China
}

\begin{abstract}
It has been well accepted by mining researchers that coal tends to undergo abrupt fracture under the coupling effect of dynamic and static loads. Hence, the study of influence of coupled static and dynamic loads on coal failure behaviour is meaningful for the understanding of coal burst. In this paper, PFC modelling of SHPB test is adopted to investigate the fracture mode and energy evolution of Australian hard coal under different combinations of pre-stress levels and impact velocities. Results have shown that high dynamic load will make the fracture mode and energy release of coal samples more violent even the static load is low. Although the strain energy increases with pre-stress level, the kinetic energy remains on a low level with the increase of pre-stress level when the impact velocity is $4 \mathrm{~m} / \mathrm{s}$.
\end{abstract}

Keywords. PFC2D; Coupled Static and Dynamic Loads; Fracture Mode; Coal Burst; Energy Evolution

\section{Introduction}

It has been widely accepted by researchers that the occurrence of coal burst is induced by the superposition of dynamic and static loads. The split Hopkinson bar (SHPB) system is a widely used high strain rate loading apparatus to determine the dynamic properties of geomaterials under complicated stress environments [1]. Li et al. modified the SHPB system to simulate the stress state of rock subject to simultaneous coupled static and dynamic loads and studied the strength characteristics and fragment size distribution of sandstone under coupled static and dynamic loads with the application of modified test system (Reinnovative testing technique of rock subjected to coupled static and dynamic loads). Besides, many scholars adopted numerical modelling software to simulate the test process and results of SHPB test of geo-materials [2-4]. The particle flow code (PFC), a type of the discrete element method (DEM), has been widely used as an approach to study the mechanical behaviour of the intact rocks in laboratory tests and engineering problems [5-7]. In this paper, the PFC modelling is extended to simulate the failure process of coal subject

\footnotetext{
* Corresponding author: tren@uow.edu.au

† Corresponding author: xy987@uowmail.edu.au
} 
to coupled static and dynamic loads produced by SHPB tests, hence, to demonstrate the contribution of dynamic load to coal burst under different pre-stress level.

\section{Methods}

\subsection{SHPB System}

As shown in Figure 1, the SHPB system generally consists of a pre-loading unit and three line up cylindrical bars named striker bar, incident bar and transmission bar, with a rock/coal sample sandwiched between the incident and transmission bar. The length and diameter of incident and transmitted bars are same with the standards established by International Society for Rock Mechanics (ISRM) [8].

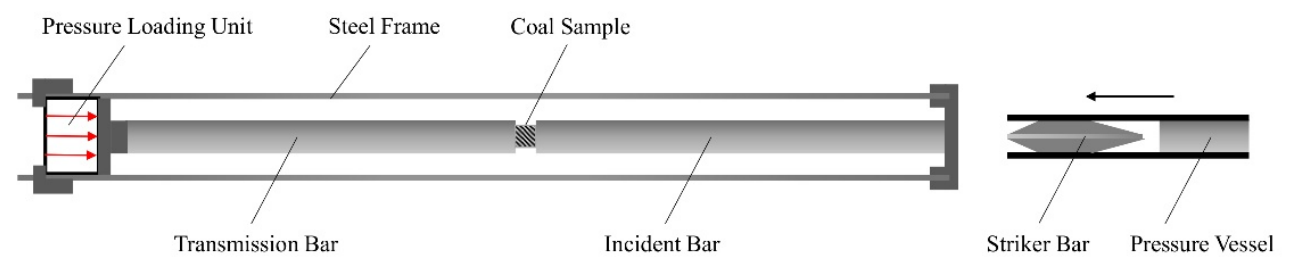

Fig. 1. Schematic Diagram of SHPB System for Coupling Loads Experiment

During the test, the coal sample will be placed between incident bar and transmission bar and then loaded with pre-compression stress by pressure loading unit (static load). The striker bar will be shot out by pressure vessel with high velocity and impacts the front surface of pressure loading unit. A compressive incident wave induced by the impact will propagate through the incident bar and reach the interface of the incident bar and coal sample. This wave will partially through the sample and then transmits into the transmission bar as a compressive stress wave, while remaining part will be reflected back into the incident bar as a tension stress wave.

\subsection{Extension of PFC modelling}

In this study, the numerical simulation of failure behaviour and energy evolution of coal under coupled static and dynamic loads includes three steps:

(1) The first step is to determine the mechanical properties of coal samples based on experiment tests. The uniaxial compression tests of coal samples were conducted in laboratory for calibrations. Calibrations including a series of trial and error numerical simulation are performed to match laboratory-measured results. The laboratory process obtained PFC microscopic parameters will be introduced in the following section.

(2) The second step is to design the test plan including stress coupling plan (pre-stress level and impact speed) and expected outcomes (energy evolution, failure behaviour and fragmentation characteristic). The impact speed of striker is based on our previous research [8] in this study.

(3) Finally, the numerical model for will be generated based on the layout of loading system, the microscopic parameters of coal, and the test plan. 


\section{Numerical Models}

\subsection{Model Parameters}

The mechanical parameters of numerical models are based on the uniaxial compression tests of coal samples taken from Australian coal seam. Coal blocks were taken from Australian coal seam with $550 \mathrm{~m}$ depth. Coal blocks are processed into $54 \mathrm{~mm}$ diameter and $108 \mathrm{~mm}$ length cylindrical samples in our laboratory by coring and cutting machine. All conditions including parallelism, flatness and verticality of coal samples should meet the standard given by ISRM. Then, the uniaxial compression tests of coal samples are conducted with the application of Instron 8033 loading machine under $0.5 \mathrm{~mm} / \mathrm{min}$ displacement control loading rate. The loading data is automatically measured by LVDT and then recorded by loading system. After the calibrations of numerical models, the obtained microscopic parameters of coal samples for PFC 2D modelling are shown in Table 1.

Table 1. Microscopic Parameters of PFC2D Models

\begin{tabular}{|c|c|c|c|}
\hline \multicolumn{2}{|c|}{ Particle Basic Parameters } & \multicolumn{2}{c|}{ Parallel Bond Parameter } \\
\hline Particle contact modules $E_{c}(G P a)$ & 1.4 & Elasticity modulus $\overline{E_{c}}(G P a)$ & 1.4 \\
\hline Stiffness ratio $k_{n} / k_{s}$ & 1.0 & Stiffness ratio $k_{n} / k_{s}$ & 1.0 \\
\hline Particle friction coefficient $\mu$ & 0.577 & Cohesion $\bar{c}(M P a)$ & 12.8 \\
\hline Particle density $\rho\left(\mathrm{g} / \mathrm{m}^{3}\right)$ & 1.4 & Tensile Strength $\overline{\sigma_{c}}(\mathrm{MPa})$ & 8 \\
\hline
\end{tabular}

\subsection{Loading Applying}

The numerical simulation of coal samples subject to combined static and dynamic loads includes two steps. The first step is to apply the static load in the axial direction with the designed stress magnitude. The second step is to generate dynamic load on statically stressed coal samples by striking incident bar with specific impact velocity. To investigate the failure mode and energy release of coal samples under different combination of static and dynamic loads, all numerical modes of coal sample are pre-loaded under different stress levels of its strength and then impacted with designed impact velocities. The selected static pre-stress level is represented by $K_{s}$ which means the ratio between pre-stress and strength of the model. The designed impact velocities of striker are $4 \mathrm{~m} / \mathrm{s}, 6 \mathrm{~m} / \mathrm{s}, 8 \mathrm{~m} / \mathrm{s}, 10$ $\mathrm{m} / \mathrm{s}$ and $12 \mathrm{~m} / \mathrm{s}$.

\section{Numerical Simulation and Discussion}

\subsection{Stress Characteristic}

As shown in Figure 2, the peak stress of coal sample increases with both pre-stress levels and impact velocities. However, the peak stress increase caused by various pre-stress levels is very obvious under low impact velocity condition while this increase is very small under high impact velocity, which means static load play an important role when the dynamic load is in small scale. 
This means that the burst risk of coal still will be high under high static load and low dynamic load as coal tend to have violent failure under high stress environment, which can be supported by previous mining practice and research. It has been demonstrated by coal burst data of many chief mining countries that the risk of coal burst or bump has obvious correlation with mining depth [9]. The possible explanation for this correlation is that the coal body bearing more pre-stress in deep area as gravitational stress is approximately liner to mining depth. It has been recovered by previous analysis that coal burst accidents occurred in Australian coalmines has no obvious correlation with strong dynamic events while the mining depth of these coalmines(around $550 \mathrm{~m}$ ) is much deeper than average mining depth (around $300 \mathrm{~m}$ ) of Australian underground mining [10].

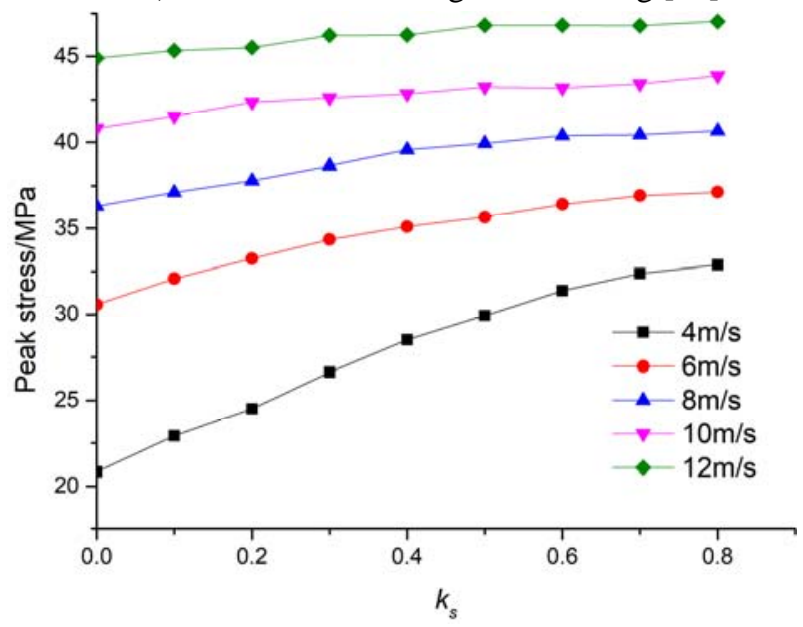

Fig. 2. Peak Stress of Coal Sample subject to Different Pre-stress Levels and Impact Speed

\subsection{Energy Evolution}

Figure 3 shows the strain energy (es) of coal samples under the different combination of pre-stress levels and impact velocities. Strain energy refers to the accumulated energy within coal sample resulting from deformation. It can be seen from Figure 3, both impact velocities and pre-stress levels have important influence on the strain energy accumulation of coal sample. However, the kinetic energy (ek) has different change trend with strain energy. Kinetic energy, which can be carried by ejected coal blocks and particles, is one of the most important parameters reflecting the intensity of coal burst event because equipment damage and personal casualties are caused by ejected coal. In Figure 4, the influence of pre-stress level on kinetic energy is very limited when the impact speed is low, which means the influence of pre-stress levels on kinetic energy release will be enhanced under high impact velocities. It also needs to be noticed that the kinetic energy value of model under 0.8 pre-stress level and $4 \mathrm{~m} / \mathrm{s}$ impact velocity is twice as much as that under 0.5 pre-stress level and $4 \mathrm{~m} / \mathrm{s}$ impact velocity, which means, even the impact speed is very low, the kinetic energy still have a comparatively rapid increase if the pre-stress level beyond $50 \%$ of its strength. 


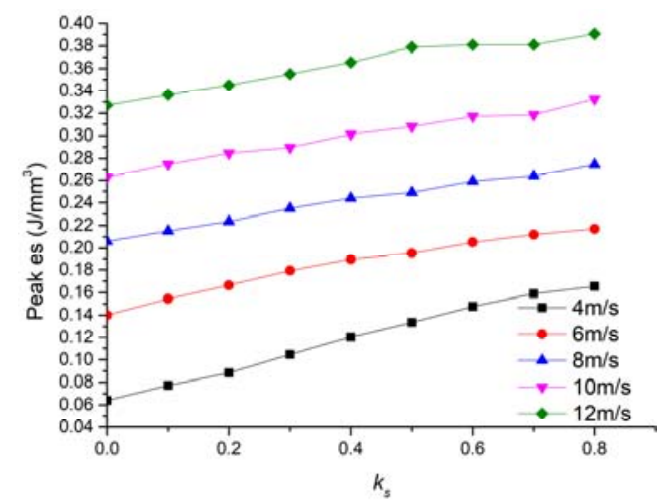

Fig. 3. Peak Strain Energy of Coal subject to Different Pre-stress Levels and Impact

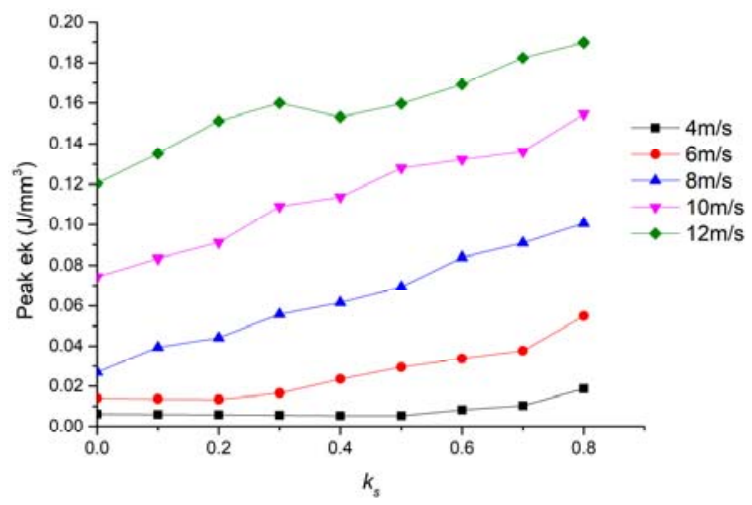

Fig. 4. Peak Kinetic Energy per unit volume of Coal Sample subject to Different Pre-stress Levels and Impact Speed

It has been mentioned by previous research that the travel velocity of coal particles can reach over $80 \mathrm{~km} / \mathrm{h}$ high during coal burst [11]. Hence, the key point of coal burst controlling is to mitigate the kinetic energy scale resulting from super-critical static loads and high dynamic loads. Generally, the dynamic load is related the instability of large geological structures including faults and hard roof. In underground mining, the static load caused by gravitational and abutment stress is very hard to be totally removed from coal body. Hence, the exploration of geological structures and seismic monitoring of instability events will be very useful for identifying the possible coal burst hazards caused by high dynamic load. Avoiding potential fault slipping by reasonable mining planning and reducing the energy intensity of roof breakage by blasting or hydro-fracturing also could be an effective way for coal burst controlling as the reduction of dynamic loads scale can obviously decrease the scale of kinetic energy.

\subsection{Fracture Mode}

As shown in Figure 5, pre-stress level has obvious influence on the failure model of coal samples when the impact velocity is low. However, in Figure 5 (b), the influence of prestress levels on fracture mode can be negligible under high impact velocity. This trend can be seen from Figure 3 and Figure 4 as well. Considering the ejection velocity and kinetic energy are positive related, Figure 5 (a) also illustrates that coal sample tend to have much more violent fracture under low impact velocity if the pre-stress level beyond $50 \%$ of its 
strength. However, as shown in Figure 5 (b), change of pre-stress levels has no obvious influence on the fracture behaviour of coal samples when the dynamic load is high.

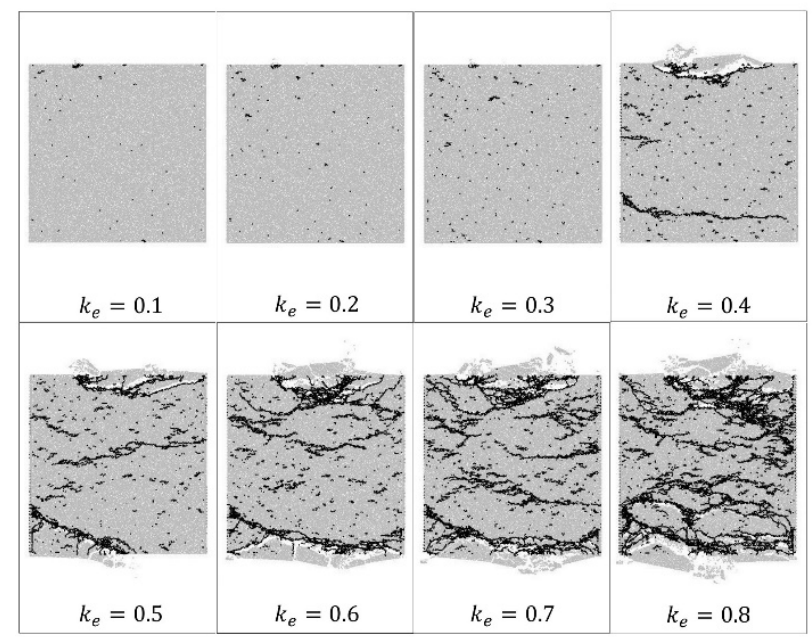

a. Failure Mode of Sample subject to Different Pre-stress Levels under $4 \mathrm{~m} / \mathrm{s}$ Impact Velocity

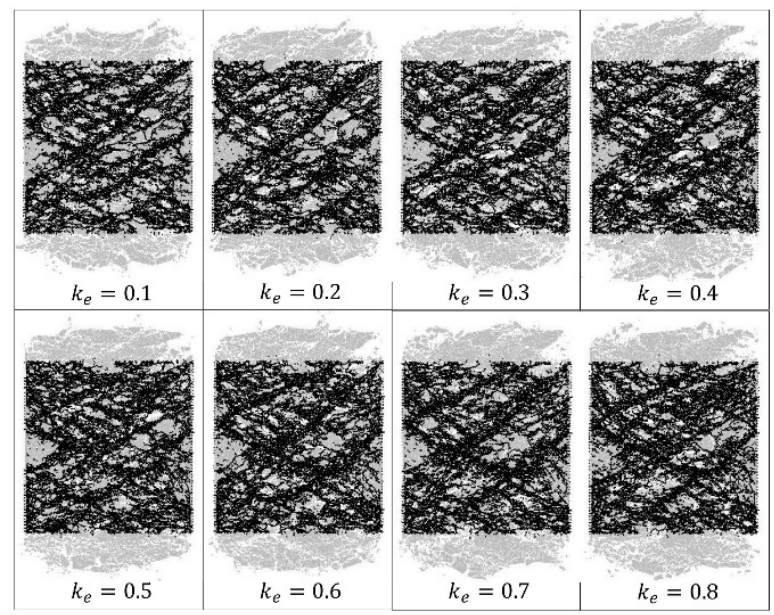

b. Failure Mode of Sample subject to Different Pre-stress Levels under $12 \mathrm{~m} / \mathrm{s}$ Impact Velocity

Fig. 5. Failure Modes of Coal Sample subject to Different Pre-stress Levels and Impact Speed

\section{Conclusions}

By adopting the PFC modelling of SHPB tests, this paper aims to study the failure behaviour and energy evolution of Australian hard coal under coupled static and dynamic loads. Based numerical results, the following conclusions can be drawn:

(1) Dynamic load has obvious influence on the peak stress of coal samples. The induced peak stress of $4 \mathrm{~m} / \mathrm{s}$ impact velocity is around $22 \mathrm{MPa}$ which is $90 \%$ of the uniaxial compression strength of the coal sample. However, coal subject to $4 \mathrm{~m} / \mathrm{s}$ impact velocity remains intact and has no obvious failure when pre-stress level is 0.1 to 0.3 . That is, the stress threshold for coal burst subject to coupled static load and dynamic loads is higher than the strength of coal. 
(2) Both impact velocities and pre-stress levels have important influence on the strain energy accumulation of coal sample. However, the influence of pre-stress levels on kinetic energy is very limited when the impact speed is low, which also means the influence of prestress levels on kinetic energy release will be enhanced under high impact velocities. Hence, the controlling of dynamic load can sharply reduce the burst risk or scale as both the influence of dynamic and static load can be mitigated.

(3) Pre-stress level has obvious influence on the fracture mode of coal samples when the impact velocity is low. As shown in Figure 5 (b), change of pre-stress levels has no obvious influence on the fracture behaviour of coal samples when the dynamic load is high. Hence, dynamic load is the key factor which contributes to the violent fracture mode as well.

\section{References}

1. K.W. Xia, W. Yao, Journal of Rock Mechanics and Geotechnical Engineering, 7, 27-59 (2015)

2. W.C. Zhu, Y. Bai, X.B. Li, L.L. Niu, Int. J. Impact Eng. 49, 142-157 (2012)

3. J.B. Zhu, Z.Y. Liao, C.A. Tang, Rock mechanics and rock engineering, 49, 3935-3946 (2016)

4. T.B. Yin, S.S. Zhang, X.B. Li, L. Bai, Engineering Fracture Mechanics, 204, 87-102 (2018)

5. Q.H. Lei, J.P. Latham, C.F. Tsang, Computers and Geotechnics, 85, 151-176 (2017)

6. J. Zhao, Y.X. Zhou, K.W. Xia, The 12th ISRM International Congress on Rock Mechanics, CRC Press, Beijing, 147-154 (2011)

7. G.F. Zhao, N. Khalili, J.N. Fang, J. Zhao, Computers and Geotechnics, 42, 1-20 (2012)

8. L.H. Tan, T. Ren, X.H. Yang, X.Q. He, International Journal of Mining Science and Technology, 28, 791-797 (2018)

9. J. Whyatt, S.P. Singh (Ed.) The 27th International Conference on Ground Control in MiningMorgantown (2008)

10. X.H. Yang, R. Ting, X.Q. He, L.H. Tan, A. Naj, K. Bob (Eds.) 2019 Coal Operators Conference, University of Wollongong, Wollongong (2019)

11. X.H. Yang, T. Ren, L.H. Tan, International Journal of Rock Mechanics and Mining Sciences, 128, 104277 (2020). 